\title{
Magnetostriction Measurements of $(\mathrm{Fe}-\mathrm{Co})-\mathrm{Mo}-\mathrm{Cu}-\mathrm{B}$ Alloys with Varying Atomic Fe/Co Ratio
}

\author{
G. Vlasák, D. JANiČKOViČ And P. ŠVEC \\ Institute of Physics, SAS, 84511 Bratislava, Slovakia
}

\begin{abstract}
Amorphous rapidly quenched ribbons of $(\mathrm{Fe}-\mathrm{Co})_{76} \mathrm{Mo}_{8} \mathrm{Cu}_{1} \mathrm{~B}_{15}$ with the ratio $\mathrm{Co} / \mathrm{Fe}=0,1 / 9,1 / 6,1 / 4,1 / 3,1 / 2,1 / 1$ and $2 / 1$ were prepared by planar flow casting. The dependence of the Curie temperature $T_{\mathrm{C}}$ on $\mathrm{Co} / \mathrm{Fe}$ ratio was determined. Due to the presence of the invar effect it was possible to measure the spontaneous volume magnetostriction in the temperature interval between $300 \mathrm{~K}$ and $T_{\mathrm{C}}$. Field dependences of magnetostriction in parallel and perpendicular directions of applied magnetic field were obtained by direct measurement method. Subsequently, saturation magnetostriction and volume magnetostriction as well as forced magnetostriction were computed. Saturation magnetostriction increases with increasing $\mathrm{Co} / \mathrm{Fe}$ ratio from 0 up to $17 \mathrm{ppm}$, depending both on the $\mathrm{Co} / \mathrm{Fe}$ ratio and on the shift of $T_{\mathrm{C}}$ with composition. The alloy with ratio $\mathrm{Co} / \mathrm{Fe}=0$ exhibits $T_{\mathrm{C}}$ near room temperature, thus field dependences of magnetostriction, corresponding to the dependences of a paramagnetic system are practically linear functions of applied field, corresponding to paramagnetic state.
\end{abstract}

PACS numbers: $75.50 . \mathrm{Kj}, 75.80 .+\mathrm{q}$

\section{Introduction}

Rapid solidification of some alloys with quenching rates up to $10^{6} \mathrm{~K} / \mathrm{s}$ can improve their physical properties or lead to new and unusual ones. This is the case of e.g. ferromagnetic rapidly quenched alloys with soft magnetic properties. Phenomenological description of the magnetoelastic coupling yields the relation for linear magnetostriction $\lambda(H)=\Delta l / l=(1 / 3) \omega(H)+(2 / 3) \lambda_{\mathrm{S}}(H)\left(\cos ^{2} \Theta-1 / 3\right)+\lambda^{\mathrm{F}}$ with $\lambda_{\mathrm{S}}$ being the saturation magnetostriction, $\omega(H)=\Delta V / V$ is the volume magnetostriction, $\lambda^{\mathrm{F}}$ is the bipolar magnetostriction (form effect) and $\Theta$ is the angle between the direction of deformation measurement and the direction of the applied magnetic field [1], where $\lambda_{\mathrm{S}}(H)=(2 / 3)\left[\lambda_{\text {par }}(H)-\lambda_{\text {perp }}(H)\right], \omega(H)=$ $\Delta V / V=\lambda_{\text {par }}(H)+2 \lambda_{\text {perp }}(H)$. The quantities $\lambda_{\text {par }}(H)$ and $\lambda_{\text {perp }}(H)$ in saturation as well as $\lambda_{\mathrm{S}}$ can be determined as in [2]. Due to the quantity $\omega$ being often not 
well defined, in technological applications the quantity $\partial \omega / \partial H$ is used instead, denoted as isotropic forced volume magnetostriction.

The present work investigates the effect of composition on magnetostriction of rapidly quenched amorphous $\left(\mathrm{Fe}_{x} \mathrm{Co}_{y}\right)_{76} \mathrm{Mo}_{8} \mathrm{Cu}_{1} \mathrm{~B}_{15}$ alloys. Compositions, where interesting values of linear, saturation and volume magnetostrictions and of forced volume and spontaneous volume magnetostrictions were expected, are reflected in the choice of $y / x=0,1 / 9,1 / 6,1 / 4,1 / 3,1 / 2,1 / 1$ and $2 / 1$.

\section{Experimental}

Samples for investigation were prepared in form of $6 \mathrm{~mm}$ wide amorphous ribbons by planar flow casting. X-ray diffraction and transmission electron microscopy were used to check the amorphous state of the samples. Measurements of field dependences of linear magnetostrictions $\lambda_{\text {par }}$ and $\lambda_{\text {perp }}$ were performed on discs with $6 \mathrm{~mm}$ diameter electrochemically etched from the ribbons using a special device [2].

The dependences $\lambda_{\text {par }}$ and $\lambda_{\text {perp }}$ (external magnetic field applied in the plane of the ribbon along the ribbon axis and in perpendicular orientation, respectively), measured on the $\mathrm{Fe}-\mathrm{Co}-\mathrm{Mo}-\mathrm{Cu}-\mathrm{B}$ system (Fig. 1) were used to compute the values of linear saturation magnetostriction $\lambda_{\mathrm{S}}$, the dependence $\omega(H)$ as well as that of forced volume magnetostriction $\partial \omega(H) / \partial H$ (Fig. 2).
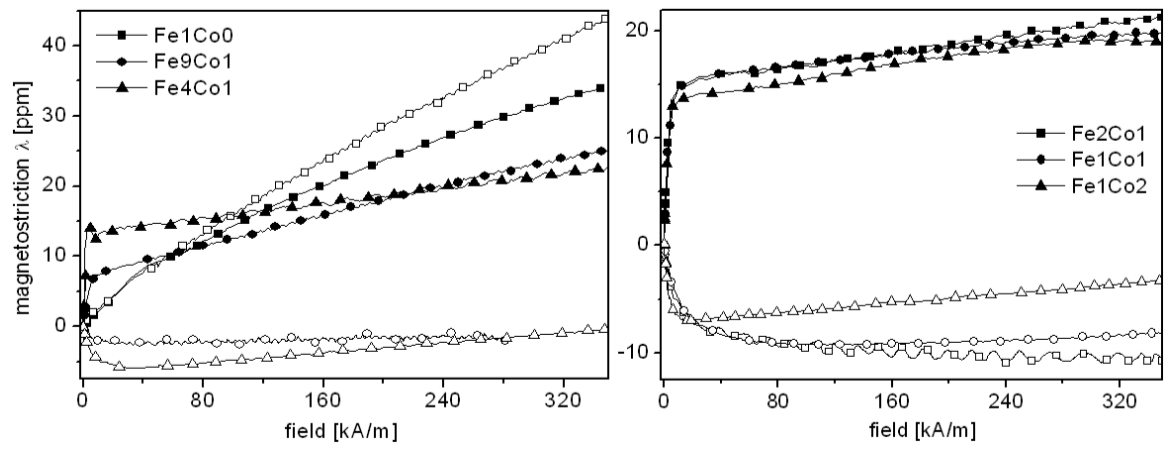

Fig. 1. Field dependence of magnetostrictions $\lambda_{\text {par }}(H)$ and $\lambda_{\text {perp }}(H)$ (full and open symbols, respectively) of $\left(\mathrm{Fe}_{x} \mathrm{Co}_{y}\right)_{76} \mathrm{Mo}_{8} \mathrm{Cu}_{1} \mathrm{~B}_{15}$ for $y / x=0,1 / 9,1 / 4$ (left) and for $y / x=1 / 2,1 / 1$ and $2 / 1$ (right).

In order to determine spontaneous volume magnetostrictions, dilatation as function of temperature was measured on a dilatometer designed for measurements on thin ribbons [3, 4]; sample length was $30 \mathrm{~mm}$. To eliminate the effect of relaxation processes, taking place in certain temperature interval below crystallization, on temperature dependences of dilatation, all samples were subjected to prior relaxation heat-treatment (Fig. 3). The Curie temperature $T_{\mathrm{C}}$ was determined from turning point of the temperature coefficient of thermal dilatation, well visible in 


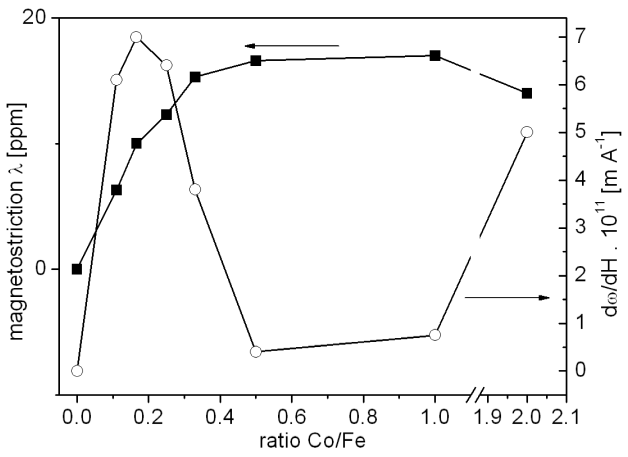

Fig. 2. Compositional dependence of saturation magnetostriction $\lambda_{\mathrm{S}}$ and forced volume magnetostriction $\partial \omega(H) / \partial H$ of $\left(\mathrm{Fe}_{x} \mathrm{Co}_{y}\right)_{76} \mathrm{Mo}_{8} \mathrm{Cu}_{1} \mathrm{~B}_{15}$ for $y / x=0,1 / 9,1 / 4,1 / 2,1$, and 2 .

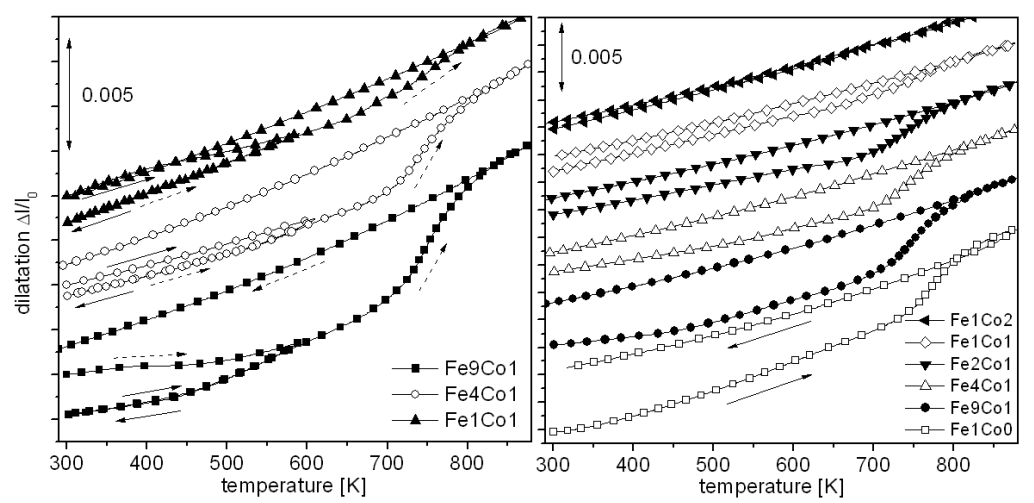

Fig. 3. Left: temperature dependences of dilatation of $\left(\mathrm{Fe}_{x} \mathrm{Co}_{y}\right)_{76} \mathrm{Mo}_{8} \mathrm{Cu}_{1} \mathrm{~B}_{15}$ during cyclic heating-cooling-heating runs (rate $5 \mathrm{~K} / \mathrm{min}$, two cycles); first cycle - relaxation annealing (solid arrows), second cycle - crystallization (dashed arrows). Right: temperature dependences of dilatation of relaxed samples (after the first heating-cooling cycle). The curves are shifted vertically for clarity.

ferromagnetic materials with invar effect. Spontaneous volume magnetostriction was determined from these dependences.

\section{Results and discussion}

The values of $\lambda_{\mathrm{S}}$ obtained from $\lambda_{\text {par }}(H)$ and $\lambda_{\text {perp }}(H)$ increase with increasing $\mathrm{Co} / \mathrm{Fe}$ ratio from 0 to $17 \times 10^{-6}$. The values obtained, measured at $300 \mathrm{~K}$, are influenced also by the value of $T_{\mathrm{C}}$; it can be expected that for the ratio of $\mathrm{Co} / \mathrm{Fe}$ $>1$ the values of the magnetostriction $\lambda_{\mathrm{S}}$ would exhibit a decreasing character, similarly as in the case of Finemets or Hitperms with higher Co content $[5,6]$.

Sample with the composition $\mathrm{Fe}_{79} \mathrm{Mo}_{8} \mathrm{Cu}_{1} \mathrm{~B}_{15}$, the $T_{\mathrm{C}}$ of which lies close to room temperature, was measured in this region. At low fields the dependences 
$\lambda_{\text {par }}(H)$ and $\lambda_{\text {perp }}(H)$ are not linear, indicating that the relation $J=\kappa H$ is not fulfilled, i.e. $\kappa$ (magnetic susceptibility) is not constant; linearity and the validity of the relation is observed for higher $H$.

Forced magnetostriction $\partial \omega(H) / \partial H$ exhibits a maximum at $\mathrm{Co} / \mathrm{Fe}=1 / 9$ and a minimum for $\mathrm{Co} / \mathrm{Fe}$ concentration ratio of $0.3-0.5$, indicating a decrease in internal stresses in the amorphous phase. The magnitude of spontaneous volume magnetostriction $\omega_{\text {spon }}$ increases with decreasing temperature from $T_{\mathrm{C}}$ down to $300 \mathrm{~K}$ and probably also further down. Relaxation processes change the bonding between magnetic dipoles. The magnitude of this magnetostriction also increases with increasing $\mathrm{Co} / \mathrm{Fe}$ ratio, as shown in Fig. 2.

The Curie temperature $T_{\mathrm{C}}$ increases with increasing content of Co from $\approx 305 \mathrm{~K}$ for Co-free alloy up to $\approx 525 \mathrm{~K}$ for $\mathrm{Co} / \mathrm{Fe}=0.25$. For alloys with higher Co content it is not possible to determine $T_{\mathrm{C}}$ by the method used. The process of relaxation and viscous flow of the sample in the vicinity of the glass transition smears out the turning point of the coefficient of thermal dilatation $\alpha$; furthermore, relaxation processes can lead to formation of different short-range ordered amorphous clusters enhancing the mentioned smearing effect.

\section{Conclusions}

Magnetostriction measurements show clearly that the ratio of concentrations of $\mathrm{Co}$ to $\mathrm{Fe}$ in $\mathrm{Fe}-\mathrm{Co}-\mathrm{Mo}-\mathrm{Cu}-\mathrm{B}$ alloys determines the concentration dependences and values of $T_{\mathrm{C}}, \lambda_{\mathrm{S}}, \partial \omega(H) / \partial H$ as well as of the spontaneous volume magnetostriction. The values of $T_{\mathrm{C}}$ and $\partial \omega(H) / \partial H$ were determined advantageously from temperature dependences of dilatation.

\section{Acknowledgments}

This work was supported by the projects VEGA 2/5096/25, APVT-99017904, APVV-0413-06, COST-031-06 (P17) and by the CEX "Nanosmart".

\section{References}

[1] E. Tremolet de Lacheisserie, J. Magn. Magn. Mater. 31-36, 1491 (1983).

[2] G. Vlasák, J. Magn. Magn. Mater. 215-216, 479 (2000).

[3] G. Vlasák, P. Duhaj, H. Patrasova, P. Svec, J. Phys. E, Sci. Instrum. 16, 1203 (1983).

[4] G. Vlasák, P. Švec, J. Electrical Eng. 55, 81 (2004).

[5] A. Kolano-Burian, T. Kulik, G. Vlasák, J. Ferenc, L.K. Varga, J. Magn. Magn. Mater. 272-276, 1447 (2004).

[6] G. Vlasák, P. Švec, D. Janičkovič, Czech. J. Phys. 54, D181 (2004). 